\title{
Beam-propagation method for analysis of $z$-dependent structures that uses a local oblique coordinate system
}

\author{
Yih-Peng Chiou and Hung-chun Chang \\ College of Electrical Engineering, National Taiwan University, Taipei 106-17, Taiwan
}

Received March 27, 1998

\begin{abstract}
In the most current beam-propagation method (BPM), the coupling between the transverse and the longitudinal fields in $z$-dependent structures is ignored under the staircase approximation, which results in violation of power conservation. We propose a novel BPM that is derived in a local oblique coordinate system to analyze $z$-dependent structures accurately and efficiently without taking the staircase approximation. The coupling between the transverse and the longitudinal fields is automatically included in the local oblique coordinate system, and power is thus conserved if the reflection is neglected. (C) 1998 Optical Society of America

OCIS codes: $260.2110,350.5500,230.7370,350.7420$.
\end{abstract}

The beam-propagation method (BPM) has been widely used in the investigation of optoelectronic devices for years and has been improved by various modifications. A comprehensive review can be found in Ref. 1. In the conventionally used BPM's, wave propagation along or near the $z$ axis is assumed, which does not correspond to the physical propagation direction of the field in the tilted-waveguide case. Wide-angle BPM's were proposed to extend the application to off-axis wave propagation., ${ }^{2,3}$ Nonetheless, to apply the current BPM's, which were developed in the rectangular coordinate system (RCS), to $z$-dependent structures, we need to make the staircase approximation. Consequently, small meshes must be employed to minimize the discretization error, which requires large memory and much computation time. Recently, BPM's based on the oblique coordinate system (OCS BPM's) were proposed $^{4,5}$ to solve tilted-waveguide problems efficiently. Since the staircase approximation is avoided and rapid field variation in the transverse directions is removed by the slowly varying approximation, the accuracy and efficiency of the OCS BPM's are much better than those of methods using rectangular coordinate systems (RCS BPM's)., ${ }^{4,5}$ However, the interfaces between the waveguide core and cladding must be parallel to the fixed OCS in the OCS BPM's, which restricts their applications, and consequently they cannot be applied to such frequently used structures as tapered or curved waveguides.

Besides, in the current RCS BPM's, derived with only the transverse fields taken into account, the derivative of the refractive index along the $z$ axis, or equivalently the coupling between the transverse and the longitudinal fields, is ignored for $z$-varying structures under the staircase approximation. ${ }^{6}$ Such coupling is not significant in most calculations, and therefore consideration of it is not vital. However, in some cases the coupling can accumulate considerably during propagation, for instance, in a whispering-gallery mode in a curved waveguide with a sufficiently small curvature for true adiabatic propagation without radiation leakage. Ignoring such coupling would result in the breakdown of power con- servation. This problem can be solved by use of the conformal transformation from the curved waveguide to a straight one or by rotation of the coordinate system such that the propagation direction always coincides with the tangent to the waveguide axis at each propagation step. ${ }^{7}$ However, the interfaces of the waveguide are required to be parallel to the new propagation direction. If the curved waveguide were tapered, the coupling would still arise because of nonparallel interfaces and could not be treated by simple rotation of the coordinate system.

To alleviate such a problem, we propose a novel BPM derived in the local oblique coordinate system (LOC BPM). Instead of using a global OCS, we define a local OCS for every sampled point, and thereafter all local OCS's are combined into a global ensemble. This proposed method not only saves numerical effort as previous OCS BPM's do but also is not restricted to interface-parallel waveguides. In particular, the OCS BPM's can be considered special cases of the LOC BPM in which the tilted angles are the same, and the RCS BPM's are those with no tilted angles. Furthermore, since the coupling between the transverse and the longitudinal fields is included automatically through the coordinate transformation, the power-conservation problem in the current BPM's is avoided.

Considering an interface with tilted angle $\theta$ from the $z$ axis, as shown in Fig. 1, we make a coordinate transformation defined by

$$
\xi=x-z \tan \theta, \quad \zeta=z
$$

such that locally the refractive-index distribution $n(x, z)=n(\xi)$ and $\partial n(\xi) / \partial \zeta=0$, and hence the staircase approximation can be avoided in the discretization. We assume that the backward field can be neglected. Instead of assuming $z$ propagation by $E(x, z)=\Psi(x, z) \exp \left(-j k_{0} \bar{n} z\right)$, we assume oblique propagation along the interface by

$$
E(x, z)=\Phi(x, z) \exp \left[-j k_{0} \bar{n}(z \cos \theta+x \sin \theta)\right]
$$




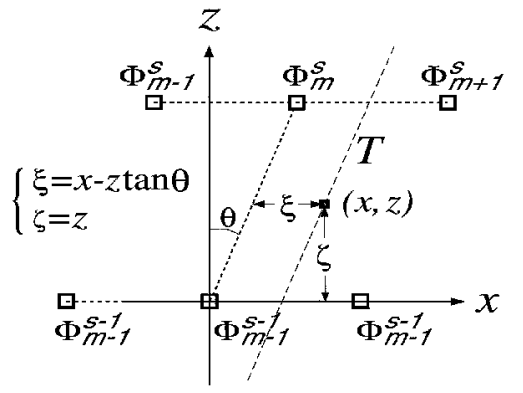

Fig. 1. Sampled points near the interface $T$.

under the slowly varying envelope approximation, and the Helmholtz equation then becomes

$$
\begin{aligned}
\left\{\frac{\partial^{2}}{\partial z^{2}}+\frac{\partial^{2}}{\partial x^{2}}-\right. & 2 j k_{0} \bar{n}\left(\cos \theta \frac{\partial}{\partial z}+\sin \theta \frac{\partial}{\partial x}\right) \\
& \left.+k_{0}^{2}\left[n^{2}(x, z)-\bar{n}^{2}\right]\right\} \Phi(x, z)=0,
\end{aligned}
$$

where $k_{0}$ is the wave number in free space and $\bar{n}$ is the reference refractive index. Note that with the slowly varying envelope $\Phi$, not only the rapid longitudinal but also the rapid transverse variations are removed, which accounts for the high accuracy and efficiency of the OCS BPM in the simulation of tilted waveguides. Making use of the coordinate transformation, we find that Eq. (3) in the local OCS is

$$
\begin{gathered}
\left\{\frac{\partial^{2}}{\partial \zeta^{2}}+\sec ^{2} \theta \frac{\partial^{2}}{\partial \xi^{2}}-2 j k_{0} \bar{n} \cos \theta \frac{\partial}{\partial \zeta}-2 \tan \theta \frac{\partial^{2}}{\partial \xi \partial \zeta}\right. \\
\left.+k_{0}^{2}\left[n^{2}(\xi)-\bar{n}^{2}\right]\right\} \Phi(\xi, \zeta)=0 .
\end{gathered}
$$

Neglecting $\partial^{2} / \partial \zeta^{2}$ and taking the central difference scheme as in the common RCS BPM's, we find that the field at the $s$ th longitudinal step and the $m$ th transverse point, $\Phi_{m}{ }^{s}$, is expressed in terms of that in the previous step as

$$
\left(M_{m}^{s} \Phi_{m}^{s}=M_{m}^{s-1} \Phi_{m}^{s-1}\right)_{L}
$$

where $L$ denotes local. Since $\Phi$ are functions of local variables in their corresponding OCS's, they are combined through Eq. (2) into the global field $E$ :

$$
\left(M_{m}^{s} H_{m}^{s} E_{m}^{s}=M_{m}^{s-1} H_{m}^{s-1} E_{m}^{s-1}\right)_{L}
$$

where the phase factor $H_{m}=\exp \left[j k_{0} \bar{n}\left(z_{m} \cos \theta_{m}+\right.\right.$ $\left.\left.x_{m} \sin \theta_{m}\right)\right]$ is restored. Combining all the sampled points, we obtain an ensemble form

$$
\left(P^{s} E^{s}=P^{s-1} E^{s-1}\right)_{G}
$$

similar to that in the RCS BPM's, where $P=$ $\left(M_{0} H_{0} M_{1} H_{1} \cdots M_{N} H_{N}\right)$ and $G$ denotes global. Equation (7) can be solved efficiently by such techniques as the Thomas algorithm. As to the truncated boundary conditions, efficient algorithms ${ }^{8}$ used in the RCS BPM can be applied.

The BPM's using the global OCS were successfully applied to solve tilted waveguides with parallel interfaces in Refs. 4 and 5. The undesirable modemismatch loss when the OCS BPM's are used can even be several orders smaller than that experienced when the RCS BPM's are used. Besides, this spurious loss when one is using the RCS BPM's accumulates to a significant amount, whereas the loss when one is using the OCS BPM's remains almost constant.

The widely used RCS BPM's suffer from an $L^{2}$ para$\operatorname{dox}^{6}$ that is due to the staircase approximation, so power conservation is usually taken as $P_{1}=\int|E|^{2} \mathrm{~d} x$. $P_{1}$ can be used in $z$-invariant structures, but we need a more rigorous definition of power, $P_{0}=\operatorname{Re} \int E^{*} H \mathrm{~d} x$, in $z$-dependent structures. To demonstrate the applicability of the LOC BPM, we consider a whisperinggallery mode with negligible radiation loss in a curved slab waveguide, shown as an inset in Fig. 2. The waveguide radius $R$ is taken to be $\left(R_{i}+R_{o}\right) / 2$, where $R_{i}$ and $R_{o}$ are the inner and outer radii at the interfaces between the core and the claddings, respectively. Note that the two interfaces are not parallel to each other for $z>0$, especially for small radii, making the equivalent waveguide width $D^{\prime}$ increase along the $z$ direction and $D^{\prime}=\left(R_{0}{ }^{2}-z^{2}\right)^{1 / 2}-\left(R_{i}{ }^{2}-z^{2}\right)^{1 / 2}=$ $\left(R_{0}{ }^{2}-R^{2} \sin ^{2} \theta\right)^{1 / 2}-\left(R_{i}^{2}-R^{2} \sin ^{2} \theta\right)^{1 / 2} \approx D \sec \theta$. Since the interfaces are not parallel, BPM's with global OCS's are not applicable, and here we resort to the local OCS.

First, we consider a waveguide with such small curvature that the field distribution is almost the same as the normal mode of a straight waveguide. The power $P_{0}(\theta)$ at any $\theta$ should always be equal to $P_{0}(z=0)$, which would lead to $P_{1}(\theta) \approx \sec \theta P_{1}(z=0)$ after the field propagates $z=R \sin \theta .^{6} \quad$ Figure 2 shows the calculated normalized power $P_{0}(z) / P_{0}(z=0)$ when the RCS BPM and the LOC BPM are used for a curved waveguide with $R=4000 \mu \mathrm{m}$, wavelength $\lambda=1.0 \mu \mathrm{m}$, waveguide width $D=2 \mu \mathrm{m}$, cladding and core refractive indices $n_{\text {clad }}=1.00$ and $n_{\text {core }}=1.03$, respectively, and under the fundamental mode incidence. Although the RCS BPM satisfies the nonphysical $L^{2}$ conservation, it gives physical power that decreases with $\cos \theta$ as the field propagates. The calculated power with the LOC BPM remains almost constant, and the small deviation is $<10^{-4}$ even after the field propagates $z=R \cos 60^{\circ}$.

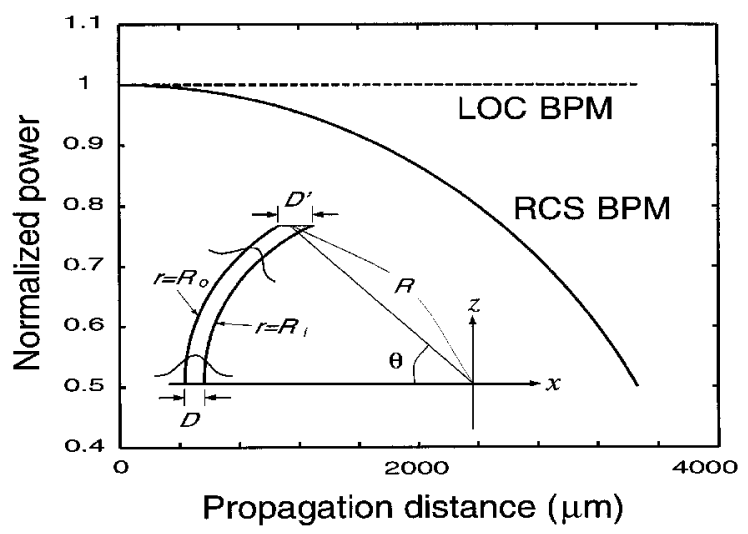

Fig. 2. Normalized power variation as the guided mode propagates along the curved waveguide. 

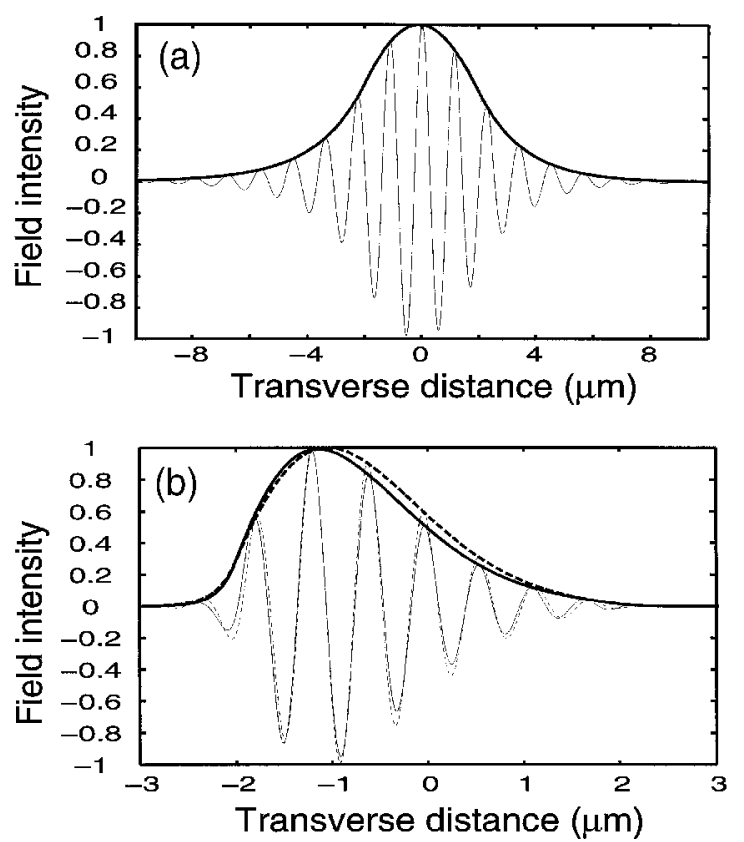

Fig. 3. Field distributions of the guided mode after it propagates (a) $z=4000 \sin 60^{\circ} \mu \mathrm{m}$ and (b) $z=25 \sin 60^{\circ} \mu \mathrm{m}$. Dashed curves, theoretical results; solid curves, results calculated with the LOC BPM. The thick and the thin curves are the field-intensity amplitude and its corresponding real part, respectively. The oscillating behavior of the real parts accounts for the transverse phase difference.

Table 1. Field-Distribution Error

\begin{tabular}{llcc}
\hline$n_{\text {core }}$ & \multicolumn{1}{c}{$R$} & $1.0-\mathrm{CR}\left(\theta=30^{\circ}\right)$ & $1.0-\mathrm{CR}\left(\theta=60^{\circ}\right)$ \\
\hline 1.03 & $4000 \lambda$ & $9.24 \times 10^{-7}$ & $6.94 \times 10^{-6}$ \\
1.03 & $2000 \lambda$ & $3.97 \times 10^{-6}$ & $3.12 \times 10^{-5}$ \\
1.03 & $1000 \lambda$ & $1.24 \times 10^{-5}$ & $1.21 \times 10^{-4}$ \\
1.20 & $100 \lambda$ & $2.32 \times 10^{-4}$ & $1.49 \times 10^{-3}$ \\
1.50 & $50 \lambda$ & $8.37 \times 10^{-4}$ & $5.85 \times 10^{-3}$ \\
2.00 & $25 \lambda$ & $2.73 \times 10^{-3}$ & $2.53 \times 10^{-2}$ \\
\hline
\end{tabular}

Figure 3(a) shows the calculated and theoretical normalized field distributions as functions of the transverse distance when the LOC BPM is used after the field propagates $z=R \sin 60^{\circ}$. The field-distribution error $\epsilon_{f}=1.0-\mathrm{CR}$ is merely $6.94 \times 10^{-6}$. CR is the cross correlation between the calculated field and the theoretical field, $\tilde{F}(x)=F(x \cos \theta) \exp (-j \beta \sin \theta x)$, where $F(x)$ is the field distribution of the incident whispering-gallery mode and $\beta$ is the propagation constant. It can be seen that not only the field intensities but also the phases are almost indistinguishable. On the other hand, the calculated CR when the RCS BPM is used is as small as $7.43 \times 10^{-7}$. Physically, the field distribution is under a phase variation $\phi=\beta \sin \theta x$ along the transverse direction. However, the calcu- lated field distribution when the RCS BPM is used is almost of equal phase, which accounts for the considerably low CR when the RCS BPM is used.

Our calculations for similar structures with different parameters also yield excellent results. Several results are given in Table 1 for different values of $n_{\text {core }}$ and $R$; the other parameters are the same as in the case of Fig. 3. To prevent radiation losses, we take $n_{\text {core }}$ to be large enough for smaller radii. For strongly curved waveguides $(R<100 \mu \mathrm{m})$, the peak of field for the whispering-gallery mode will be noticeably shifted to the outer interface, and thus the field distribution is asymmetric. Figure 3(b) shows the calculated and the theoretical field distributions for $R=25 \mu \mathrm{m}$ and $\theta=60^{\circ}$. Theoretically, the propagation constant $\beta$ varies with $1 / r$, where $r$ is the radius shown in Fig. 2, because $\partial / \partial \theta=-j r \beta$ is constant for the whisperinggallery mode with negligible loss. If $R$ is small, the propagation constants at the inner and the outer interfaces will be noticeably different, which accounts for the larger $\epsilon_{f}$ for smaller radii and the larger field deviation farther away from the waveguide center. The LOC BPM can also be applied to other $z$-dependent structures, such as tapered waveguides.

In summary, we have developed a novel BPM derived in the local oblique coordinate system to treat $z$-dependent structures. The staircase approximation is avoided in this method, so more-accurate results than those of other methods are obtained. The powerconservation problem suffered in the conventionally used BPM is alleviated. This proposed method was numerically validated by examination of the field distribution and the power conservation for whisperinggallery modes in curved waveguides.

This study was supported by the National Science Council of the Republic of China under grant NSC872215-E-002-008.

\section{References}

1. D. Yevick, Opt. Quantum Electron. 26, S185 (1994).

2. G. R. Hadley, Opt. Lett. 17, 1426 (1992).

3. Y. P. Chiou and H. C. Chang, in 11th International Conference on Integrated Optics and Optical Fibre Communication (Institution of Electrical Engineers, London, 1997), Vol. 2, p. 19.

4. J. Yamamuchi, J. Shibayama, and H. Nakano, in Integrated Photonics Research, Vol. 13 of 1994 OSA Technical Digest Series (Optical Society of America, Washington, D.C., 1994), p. 19.

5. P. Sewell, T. Anada, T. M. Benson, and P. C. Kendall, Microwave Opt. Technol. Lett. 13, 24 (1996); J. Lightwave Technol. 13, 688 (1997).

6. C. Vassallo, J. Opt. Soc. Am. A 13, 761 (1996).

7. P. Danielsen and D. Yevick, J. Opt. Commun. 4, 94 (1983).

8. C. Vassallo and F. Collino, J. Lightwave Technol. 15, 1958 (1997). 